\title{
Acute Pericoronitis And The Position Of The Mandibular Third Molar In Nigerians
}

\author{
Akpata O.
}

\begin{abstract}
:
This study evaluates the relationship of acute pericoronitis to the position of the mandibular third molar in Nigerians. One hundred and thirty-two cases of acute pericoronitis seen over a period of 6 months at the state Dental Centre, Kaduna were studied. The tooth with the highest risk for acute pericoronitis was found to be vertically positioned third molar (57.4\%); followed respectively by distoangular (25.9\%) and mesioangular (14.8\%) impactions. The height of such a tooth in relation to the second molar was found to correlate with deep pocket distal to the tooth $(\mathrm{P}<0.05)$. The observation in this study showed that most of the teeth of the vertically impacted group were either at the same occlusal level or slightly above. A change in the classification of vertical impaction into 'false' and 'true' vertical impactions is suggested.
\end{abstract}

\section{INTRODUCTION:}

Pericoronitis is a painful debilitating condition, which is most commonly found in young adults ${ }^{1}$. John Hunter ${ }^{2}$ recognized its effect when he said that 'nothing but the removal of the tooth or teeth will remove the evil in many cases. Henry ${ }^{3}$ reviewed the disastrous consequences of pericoronitis in the pre-antibiotics days and advocated their removal. It is the most common symptom ${ }^{4}$ and indication ${ }^{5}$ for impacted third molar extraction.

KEY WORDS: Acute-pericoronitis, impactionposition, frequency

Department of Oral Surgery and Oral Pathology, Faculty of Dentistry, University of Benin City, Nigeria cbnosagie@yahoo.com
Studies relating the type of impaction to the frequency of pericoronitis are few. Wallace ${ }^{6}$, Leone et $\mathrm{al}^{7}$ and Lee et $\mathrm{al}^{8}$ in their studies reported the preponderance of vertically impacted third molars, while Kay ${ }^{9}$ showed that more than one third of the total cases involved were mesioangular impactions. Acute and subacute pericoronitis as defined by Kay $^{9}$ were combined together. Acute pericoronitis was defined as sharp or throbbing pain, redness, swelling and or purulent discharge associated with the third molar. Limitation of opening, discomfort during swallowing, fever, lymphadenopathy, bad breath when present during the episode was recorded.

A Medline search using different phrases and personal searching of five recent 
references on closely related subjects produced no previous reports, that relates the type of lower third molar impaction with the frequency of acute pericoronitis in Nigerians

The purpose of this study is to evaluate the relationship of the type of impaction of the lower third molar to the frequency of acute pericoronitis in Nigerians.

\section{METHODS:}

One hundred and thirty-two (132) cases of acute pericoronitis seen over a period of 6 months at the state Dental Centre, Kaduna were studied. 24 of these cases could not be further analyzed because of loss of radiographs.

All subjects were examined with mouth mirror and sickle probe in a dental chair using artificial light. Intraoral periapical radiographs were taken. The position of the impacted tooth was determined by means of the method first described by George Winter10. The height was determined as follows, from the radiographs:

(A) Above the occlusal plane: the crown of the third molar was above the occlusal plane.

(b) The crown of the third molar was at the occlusal plane. (c) Below the occlusal plane: the crown of the third molar is below the occlusal plane.

Data from all cases of pericoronitis was entered into a prepared form and the analysis was done by a statistical package for microcomputers using:

(a) t- test

(b) Chi-square test

(c) Correlation coefficient $\mathrm{p}$ values less than $0.05(\mathrm{P}<0.05)$ were taken to imply statistical level of significance.

\section{RESULTS:}

One hundred and thirty-two (132) cases of acute pericoronitis were seen during the period of study. The ages of the patients ranged from 16-45 years and there were 64 females and 68 males, with female to male ratio of $1: 1.77 .3 \%$ of patients were between ages $16-25$ years, with $39.4 \%$ between $21-25$ years and $37.9 \%$ between $16-20$ years.

Twenty-four (24) cases could not be further analyzed because their radiographs were not available. Of the remaining 108 cases of acute pericoronitis, 62 cases (57.4\%) were vertically impacted, 28 cases $(25.9 \%)$ were distoangular, 16 cases (14.8\%) were mesioangular and 2 cases (1.9\%) were horizontal impaction. 
43 Journal of Medicine and Biomedical Research

Table I: Position of mandibular third molar and age.

\begin{tabular}{lllllll}
\hline Position & & \multicolumn{2}{c}{ Age (years) } & & \\
& $16-20$ & $21-25$ & $26-35$ & $36-40$ & $41-45$ & Total \\
\hline Vertical & 29 & 24 & 7 & 1 & 1 & 62 \\
\hline Distoangular & 8 & 8 & 10 & 2 & 0 & 28 \\
\hline Mesioangular & 7 & 4 & 5 & 0 & 0 & 16 \\
\hline Horizontal & 1 & 1 & 0 & 0 & 0 & 2 \\
\hline Missing cases & 5 & 15 & 3 & 1 & 0 & 24 \\
\hline Column total & 50 & 52 & 25 & 4 & 1 & 132
\end{tabular}

Table 1 is a cross tabulation between impaction and age of the patients. Of the 62 cases of vertical impactions seen, 47 were associated with deep pockets (Table 2). With regard to the height, 17 cases (36.2\%) were above the occlusal plane and one below the plane.

Table II: Position of mandibular third molar and deep pocket

\begin{tabular}{llll}
\hline $\begin{array}{l}\text { Position of } \\
\text { Third molar }\end{array}$ & $\begin{array}{l}\text { Presence of } \\
\text { deep pocket }\end{array}$ & $\begin{array}{l}\text { Absence of } \\
\text { deep pocket }\end{array}$ & Total \\
\hline Vertical & 47 & 15 & 62 \\
\hline Distoangular & 17 & 11 & 28 \\
\hline Mesioangular & 1 & 15 & 16 \\
\hline Horizontal & 0 & 2 & 2
\end{tabular}

There was a significant correlation between the height of impacted tooth in relation to the second molar and the deep pocket distal to the tooth $(\mathrm{P}<0.05)$.

DISCUSSION:

Pericoronitis, an acutely painful condition is found associated with various patterns of mandibular third molar impaction in young adults ${ }^{1-9}$. There was no sex difference observed in pericoronitis in this study. This agrees with other findings Kay ${ }^{9}$, Wallace $^{6}$ and Lee et $\mathrm{al}^{8}$. In Kay's word, it would seem to indicate that in a modern society despite sexual disparity in the jaw size, sex differences are not important in the development of pericoronal flap infection. Rather, it can be attributed to the oral health status of the affected individual.

Although patients at any age can present with acute pericoronitis, susceptibility to this 
infection was greatest between 16-25 years with a maximum incidence between 21-25 years. Lee et $\mathrm{al}^{8}$ observed in their study that pericoronitis of the mandibular third molar was frequently seen in the third decade. This could be due to some other causative factors involved in pericoronitis, such as erupted maxillary third molar and deep pocket, which are very active during this period.

There was an incidence of vertical impaction of $54.4 \%$, followed by distoangular impaction of $25.9 \%$ and mesioangular impaction of $14.8 \%$ in this study (Table 1 ). Because of the difficulty in classifying borderline distoangular impactions, some investigators classify vertical and distoangular impactions together. Wallace 6 had $90 \%$ of cases of pericoronitis associated with vertically inclined third molars. There was no mention of distoangular impactions and criteria for his classification were not reported. If borderline distoangular impactions are classified as vertical, then the studies by Wallace ${ }^{6}(90 \%)$, Halverson et al $(80 \%)^{11}$ and Leone et $\mathrm{al}^{7}(84 \%)$ agree with the present study, which has a combined percentage of $83.3 \%$. The combined percentage in Kay's ${ }^{9}$ studies was $58.8 \%$. It appears from these studies that the vertical or borderline distoangular impaction has the highest risk for pericoronitis in the presence of other causative factors. The difference in incidences of mandibular third molar impaction recorded by various authors, especially Kay's findings ${ }^{9}$ compared with our study may be related to the different anthropological profiles of the races. The length of the mandibular body and ramus accounts for this. Since the occurrence of third molar impactions have been quoted to range from $9.5 \%$, Helleman ${ }^{12}$ to $20.0 \%$, Bjork etal $^{13}$; a greater percentage (80\%-90\%) will be expected to erupt into the oral cavity. According to Schuller ${ }^{14}$ a 'zero tilt' does not necessarily imply a healthy clinical condition. As long as the causative factors of pericoronitis are present, it will likely affect the $80 \%-90 \%$, which erupt into the oral cavity.

Since vertical impactions have the highest risk of pericoronitis, it was reviewed for the presence of deep pocket and the tooth height in relation to the second molar in this study. A deep pocket was present in 65 cases whose third molar region showed a distal pocket radiologically (Fig 1), Table 2. Of these, 47 cases were associated with vertical impaction, 17 cases with distoangular and one case with mesioangular impactions. This agrees with Kay's ${ }^{9}$ study, which had a combined percentage of $99 \%$ of deep pocket affecting the vertical and distoangular impacted third molars.

For the height in relation to the occlusal plane of the second molar, 17 cases were above the plane, 29 cases at the same occlusal level and one below the occlusal plane. This result is similar to that of Leone et $\mathrm{al}^{7}$ and wallace6 who described the tooth at the highest risk for pericoronitis as a 'fully erupted vertical impacted mandibular third molar that is in contact with the adjacent teeth. There was a correlation between the height and the deep pocket, which was statistically significant $(\mathrm{P}<0.05)$.

What that means in clinical terms is that 'the higher eruptive level of the wisdom tooth raises the retromolar papilla or pericoronal flap into actual contact with the cusps of the opposing molar during mastication causing trauma. This could result in frequent pounding of the retromolar papilla causing repeated infection and consequently a deep pocket formation. These two important causative factors in the vertically impacted tooth might contribute to the high frequency of risk for pericoronitis.

The observation that most of the teeth of the vertically impacted group were either at the same occlusal level with the second molar or slightly above brings into question whether the designation of such teeth as 
impacted was appropriate. Kay ${ }^{9}$ must have wondered at such categorization when he wrote about 'mandibular third molar designated as such, because of the intervention of occlusal gum flap. Tetsch ${ }^{15}$ definition of impacted tooth is 'a tooth prevented from erupting by adjacent hard structure (teeth, tumours) or embedded in bone on all sides'. Other definitions include the prevention from eruption by soft tissues, Archer ${ }^{16}$. Eruption is generally understood by Bhasker $^{17}$ as axial or occlusal movement of tooth from its developmental position in the jaw to its functional position in the occlusal plane. From this definition most of the vertically positioned teeth in this study are not impacted.

In order to eliminate the confusion in terminology and yet retain the word impaction (because old habits die-hard), it is suggested that such teeth be termed false vertical impactions, while the others be termed true vertical impactions'. The need for this change in designation is in the treatment. Fully erupted or over-erupted mandibular third molars can be extracted like any other teeth in the mouth with forceps or elevators. This is in contrast to true vertical impaction where the obstruction may have to be removed surgically; following the National Institute of Health consensus criteria,18 before third molar surgery should be carried out. The consensus criteria are: recurrent pericoronitis, caries not amenable to restorative measures, dentigerous cyst, internal or external root resorption and periodontal disease to which the third molar was contributing.

\section{References:}

1. Rub J. Removal of impacted lower third molar with acute pericoronitis and necrotising gingivitis. Br. J Oral Surgery. 1970; 7:153-160.
2. Hunter J. The natural history of the human teeth; explaining their structure, use, formation, growth and disease, London 1771.

3. Henry C.B. Wisdom teeth and their complications. Lancet, 1935; (i) 313316.

4. Obiechina AE, Arotiba JT, Fasola AO. Third molar impaction: evaluation of the symptoms and pattern of impaction of mandibular third molar teeth in Nigerians. Odontostomatol Trop. 2001 Mar;24(93):22-5.

5. Bataineh AB, Albashaireh ZS, Hazza'a AM. The surgical removal of mandibular third molars: a study in decision-making. Quintessence Int. 2002 Sep;33(8):613-7.

6. Wallace S.R. Pericoronitis and Military Dentistry. Oral Surg Oral Med Oral Path. 1966; 22:545-547.

7. Leone S.A, Edenfield J.J, Cohen M.E Correlation of acute pericoronitis and the position of the mandibular third molar. Oral Surg Oral Med Oral Path. 1986; 62:245-250.

8. Lee DK, Kim BJ. The relation of pericoronitis to the position of the mandibular third molar. [Article in Korean]: Taehan Chikkwa Uisa Hyophoe Chi. 1989 Feb; 27(2): 201-9.

9. Kay L.W. Investigation into the nature of pericoronitis. Br J Oral Surg. 1966; 3:188 - 205.

10. Winter G.B. Impacted Mandibular third molar. Medical Book Co., 1924.

11. Halverson BA, Anderson WH 3rd. The mandibular third molar position as predictive criteria for risk for pericoronitis: a retrospective study. Mil Med. 1992 Mar;157(3):142-5. 
12. Hellmann M. Some aspects of wisdom teeth and their importance. Arch. Clin., Oral Path. 1938;2.125.

13. Bjork A, Jensen E, Palling $M$. Mandibular growth and third molar impaction. Europ. Orthodont. Sve. Trans. 1938; 163.

14. Schuller W.R. Positional ahnges in mesioangular impacted third molars during a year. J. An Dent. Assoc. 1979; 99: 460-464.

15. Tetsch, Wagner. Operative extraction of wisdom teeth. Wolf Medical Publications Ltd. 1985; 9.
16. Archer W.H. Oral and Maxillofacial Surgery Vol.1 Fifth Edition. W.B Saunders Publishing Company. 1975; 250-310.

17. Bhasker S.N Orban oral histology and embryology. 8th Edition. St. Louis: C.V Mosby Co., 1976; 361.

18. National Institute of Health. Consensus development conferences for removal of third molars. J. of Oral Surg. 1980; 38: 235-6. 